empyema for two reasons-(I) If anything were to go wrong with the pump, water would pass into the cavity; (2) if there were much discharge the pump would become dirty and refuse to act. A large bottle was connected between the pump and the chest, and this was partially filled with carbolic lotion; by placing the bottle on a higher level than the pump, it was found that even when the water was flowing extremely slowly, respiratory movements did not lead to sufficient alteration in pressure to draw the water into it. A negative pressure of $80 \mathrm{~mm}$. Hg was used in adult cases, and this was accomplished by having a fall of nearly 2 metres.

Since the apparatus described above, although possessing the advantage of costing less than ten shillings, nevertheless labours from the disadvantage of requiring a slight amount of attention, it was thought worth while to devise one that would work without any attention at all. A small exhaust pump of about 16 c.cm. capacity was run by a small electric motor; with this arrangement it is necessary to place a valve in the system to prevent too great a negative pressure being developed. The arrangement which was adopted consisted in connecting the pump with a large wide-mouthed bottle, through the cork of which three tubes passed. One of these was connected with the pump, one with the chest, and the third was open above, but inside the bottle passed into a cylinder containing mercury.

The depth of the mercury was over $15 \mathrm{~cm}$., the tube could be drawn up so that the distance of the end from the surface of the mercury could be adjusted with ease. It is necessary for the cylinder to be at least 2 centimetres in diameter, because air bubbling through mercury agitates it so greatly that if a small cylinder be used the mercury would be thrown out of it; gauze should be tied over the top of the cylinder. The negative pressure developed depends upon the depth of the opening of the tube below the surface of the mercury and efficiently prevents a dangerous exhaust being established.

The treatment is painless; usually the pulse rate is slightly accelerated, but that may be due to the excitement of having the apparatus by the bedside.

\section{The Advantages.}

The advantages which may be claimed for this treatment are two: the negative pressure tends to expand the lung without necessarily causing emphysema of the other lung, which may be expected from the use of James's bottles.

The negative pressure produces a hyperaemia of the tissues of the pleural cavity, which probably increases their local resistance.

The comparative opsonic values of exudates from the pleurae under normal and under reduced pressures are being estimated.

This is only a preliminary account, but the results obtained so far seemed to me to justify publication.

\section{IRON ACETATE IN THE TREATMENT OF PNEUMONIA. \\ By HERBERT J. ROBSON, M.R.C.S., L.R.C.P.,} Leeds.

For the last seven years all cases of pneumonia-whether bronchopneumonia or lobar pneumonia-met with in $\mathrm{my}$ practice have been treated by iron acetate, and in severe cases by alternate doses of iron acetate and strychnine, and with surprisingly good results. The statistics I intend publishing later when I get more time at my disposal.

A furred tongue or complications such as bronchitis and pleurisy or whooping-cough seem to make no difference to the good effect of the treatment, though the lower the condition of the patient the better is the value of the treatment demonstrated. The prescriptions used are:

$\mathrm{R}_{\mathrm{B}}$ Liquor forri perchlor. $m \times \mathbf{x}$, liquor ammon. acetat. $31 \mathrm{j}$, aq. chlorof. ad $38 s$ (adult dose). Take every 4 hours in water when given alone : take every 6 hours alternately with the strychnine mist. when this latter is needed. (It is administered every 6 hours until the patient is well over the crisis ; then it is given every 8 hours, and later every ${ }_{2}$ hours alternately with the strychnine.)

$\mathrm{B}_{\mathrm{R}}$ Liquor strychninae $\mathrm{mv}$, aq. chlorof. ad jss; fl. mist. Take every 6 hours in water alternately with the ferri mist. and as above stated (in severe cases only).

$R_{*}$ Pine oil, for dry inhalation on a clean handkerchief.

Alcohol is seldom given until towards or after the crisis, and often it is not required at. all, and then only as the pulse or condition of the patient directs.
Oxygen is seldom required; and, in my opinion, its value is very much overestimated. But when it is given it is administered "warm," being either gently blown into the patient's mouth and nose at a temperature of $90^{\circ}$ or $95^{\circ} \mathrm{F}$., at which temperature there is no cold dranght or feeling of cold, or inhaled directly at a temperature of $65^{\circ} \mathrm{F}$. from the oxygen warmer made for me by Messrs. Reynolds and Branson; though always the temperature of the oxygen is regulated by the warmer.

Quinine is never given, as in my opinion it does positive harm in this disease.

Digitalis seems seldom needed if the treatment by iron acetate is adopted early.

Antipyretics of the coal-tar series are carefully avoided, as in this disease I believe them to be dangerous.

Tepid sponging or the cold pack is resorted to for lowering a high temperature; though under the above treatment it seems to be seldom required. The temperature of the bedroom is kept at $63^{\circ} \mathrm{F}$., and free ventilation is encouraged.

For pleuritic pain an occasional mustard leaf and a flannel binder carefully applied round the chest are resorted to. If these fail to relieve the pain, a small dose of morphine is given. Systematic poulticing is condemned.

For insomnia, which is seldom met with, trional is given, $15 \mathrm{gr}$. , repeated in six or eight hours if needful.

\section{Remarks.}

The treatment by this alternate administration of the iron and the strychnine (where the latter is called for) seems to be particularly useful under the following conditions :

I. Cases of severe bronchopneumōnia occurring in infants or children and in catarrhal and lobar pneumonia occurring in debilitated subjects.

2. Where one has not got two properly-trained nurses for night and day respectively, as by the regular administration of the strychnine through the night as well as through the day, the heart's action is kept up, and the danger of heart failure at the crisis is very much lessened.

3. In that infectious, creeping, and spreading form of pneumonia following iniluenza.

4. The crisis is hastened and favourably modified, the virulence of the disease is lessened, and the complication of empyema seldom occurs.

5. Even when bronchitis is present, there seldom seems need to order depressing drugs like ipecacuanha, as the iron acetate seems to act as a good expectorant.

\section{NEW METHODS OF STUDYING AFFECTIONS OF THE HEART. \\ Bx JAMES MACKENZIE, M.D., Burnley.}

\section{V.-THE INCEPTION OF THE RHYTHM OF THE} HEART BY THE VENTRICLE.

Is this series of papers my main object has been to demonstrate the method of investigating the movements of the heart. For this purpose I have used for illustration cases where digitalis has modified; the functions of the heart muscle, as thereby we can the more readily appreciate the nature of the changes, and more detinitely determine the manner of their production. There are, however, many forms of heart failure in which digitalis has little or no effect, and, truth to tell, where no remedy seems capable of checking the onward progress of the heart failure. But even from the fact that these hearts do not sensibly react to digitalis, certain limited deductions may be drawn. Of such forms of heart failure there is one to which I have been giving special attention for many years, and the treatment of which has been unsatisfactory. This form includes many of those cases of heart failure with enlargement of the organ and a continued irregularity in its action, often without any antecedent history of valvular affection, and in whom postmortem examination reveals no structural changes in the valves. Much has been written concerning such cases, and numerous terms of supposed diagnostic significance have been applied to them, such as chronic myocarditis, fatty degeneration, dilated heart, paroxysmal tachycardia, delirium cordis, chronic bronchitis with irregular heart, and so forth. A careful study of such cases-and particularly of those, now somewhat numerous, whom I have seen start on their downward career-convinces me that the whole train of 
oymptoms is due to the inefficient action of the heart, induced by the inception of the rhythm of the heart by the ventricle. The manner in which this ventricular rhythm starts varies, in some cases gradually, by the occurrence in increasing frequency of ventricular extra-systoles, in some cases suddenly in consequence of unwonted exertion, while in others the heart quietly drops into this condition without apparent cause. In all cases the heart's work is performed less efficiently, and usually there is at once a great limitation of the field of cardiac response-the patients cannot exert them. selves with their wonted ease and comfort, shortness of breath, palpitation or pain being easily induced.

In certain cases, within a few hours of the inception of the ventricular rhythm, especially where the heart's action is rapid, marked changes take place in the circulatory system. The lips become darker in hue, and the face dusky. The area of the heart's dullness increases, the respirations become short and hurried, marked pulsation in the neck occurs, due to waves of blood being thrown back into the jugular veins. Then speedily follows oedema of the lungs and legs, enlargement of the liver, and all the typical signs of grievous heart failure. Other cases may not show such symptoms so rapidly; some, indeed, show but a slight limitation of the field of cardiac response, and may for years live a quiet life with continued irregularity in the neart's action, but liable to temporary attacks of heart failure.

When by some good fortune the heart reverts to its normal rhythm, the improvement in the patient's condition is at once remarkable, and the rapidity with which all the signs of heart failure disappear is even more striking than the rapidity with which they came on. In this fact-the restoration of the heart to the normal rhythm-we have the objective towards which all our efforts at treatment ought to be directed. Inasmuch as probably nearly one-tbird of the cases of serious heart failure we are called upon to treat owe their suffering to the inception of the rhythm by the ventricle, it will be realized that such an inquiry as this is not of mere academic importance, but is a prime necessity if we desire to treat our patients intelligently.

The Cause of the Ventricular Rhythm is due to OperExcitability OF the Fibres JOINING AURICLE AND VENTRICLE.

In order that the aim for diagnostic and therapeutic purposes might be precise and definite, I have for many years been collecting evidence which might throw light upon the subject. The facts that I have gathered point to the conclusion that the cause of the ventricles contracting before the auricles ("ventricular rhythm") is due to an over-excitability of the muscular fibres joining the auricle and ventricle. I am aware that the evidence I shall give is not absolutely conclusive; still I shall adduce sufficient presumptive proof in favour of this suggestion, and in doing so try to indicate the lines on which investigation into this difficult problem may profitably be pursued.

\section{EXPERIMENTAL Evidence.}

Although I have worked out the solution of this question from the strdy of the action of the human heart, I find many striking corroborative and suggestive facts given by experimental physiologists, more especially in the researches of Gaskell. This observer specially insists on the fact that the muscular fibres joining auricle and ventricle in cold-blooded animals are extremely sensitive to stimulation. "Touch the auriculo-ventricular ring with the slightest stimulation, immediately a series of rhythmical contractions occur. It is most striking to see, after removal of the septum, how every portion of auricular and ventricular tissue can be explored up to the very edge of the ring without obtaining more than a simple contraction, while immediately the needle touches the muscular ring a series of rapid contractions results." He points out that in applying a ligature or section in the auriculo-ventricular groove a series of contractions immediately results from the stimulation of these fibres. Stimulation of the vagus sufficiently strong to stop the auricular movements may be followed by a starting of the heart's action, due to the ventricle taking on the rhy thm - the rhythm arising at these $a \cdot v$ fibres.

From such results as these one can safely deduce that there are two places in the heart where there is an exceptional tendency for the rhythm of the heart to start-at the sinus venosus (in man at the mouths of the great veins and adjacent portions of the auricle) and at the canalis auricularis (in man in the muscle fibres joining auricle and ventricle).
Normally the rhythm starts at the former place, but when any undue excitation affects the latter, or even when the former fails to transmit a stimulus to the ventricle, the rhythm of the heart may start at these $a-v$ fibres.

\section{The Nature and Prace of Origin of the Ventricular} ExTra-SYsTOLK.

By the following train of reasoning I now suggest that the starting point of what I have hitherto called the "ventricular rhythm" is at the fibres joining the auricle and ventricle. In setting forth this argument I would specially call attention to the utility of this method of observation by pointing out the fund of information to be obtained from the study of such a simple tracing as Fig. 2 .

At the Nottingham meeting of the British Medical Association in 1892 I demonstrated the fact that in certain common forms of irregular heart, when the pulse seemed to miss a beat the irregularity was due to the contraction of the ventricle prior to and independent of the auricle (Fig. I). The early occurring ventricular contraction-which I then called the ventricular extra-systole-may cause so small a radial beat as to be imperceptible to the finger although shown in a sphygmograph, as in Fig. 1. I published the proofs for this statement in the Journal of Pathology in 1894.

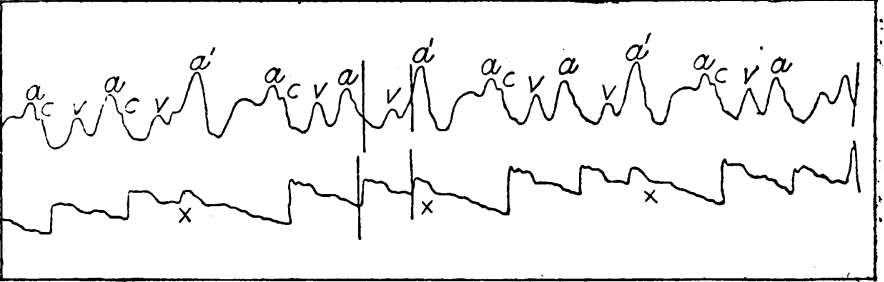

Fig. r.-Simultaneous tracings of the jugular and radial pulses. $\left(a\right.$ and $\left.a^{\prime}\right)$ radial pulse is irregular, the waves in the jugular (a and $x$ in the ris auricular ventricle has contracted prior to and independent of the stim the from the auricle.

Though this statement passed unnoticed by clinicians and physiologists, I was convinced that in it lay the key to many of the mysteries of the heart's varied action, and I have never cessed to inquire deeper into the true meaning of this observation. Subsequently Engelmann published the results of his experimental observations on the extrasystole in the frog's heart, and gave a detailed explanation of the meaning of its chief characteristics. Following on Engelmann's observations, iVenckebach found extra-systoles in man, and Cushney observations, Wenckebach found extra-systoles in man, and Cushney extra-systole in the mammal heart, and subsequently these observers described the same irregularity in man, depending, however, for their proof only on the fact that the irregular period, including the extrasystole, occupied two pulse periods, wheress in my original paper I showed the actual relationship of the auricular systole to the ventricular, as in Fig. $x$.

I have in previous papers called attention to the fact that a longer rest after systole permits the conductivity of the $a \cdot v$ fibres to be more completely restored, as shown by the duration of the $a-c$ interval. When the conductivity is normal, a quickening of the heart beat or a slowing has little apparent effect upon the duration of the $a \cdot c$ interval. But if there be the slightest depression of this function, the period of rest has a very marked influence, so that in the duration of such $a \cdot c$ intervals we have a standard by which we can approximately estimate the time of the previous stimulation of these fibres. In Fig. 2 an extra-systole is shown at $x$ in

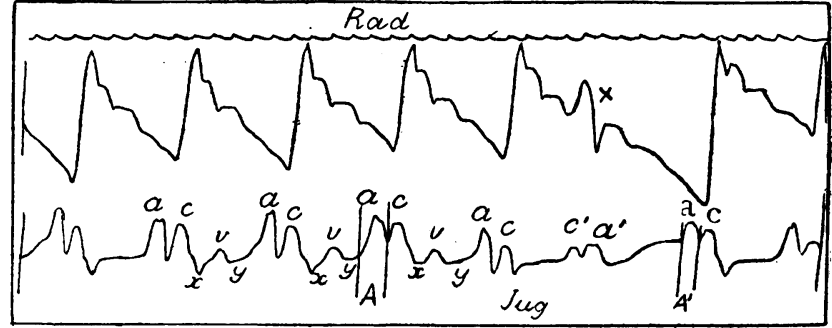

Fig. 2.-Simultaneous tracings of the radial and jugular pulses, showing the occurrence of a ventricular extra-systole at $x$ in the radial tracing; $c$ corresponds in time with $x$, and is due to the occurring at the normal time, and after the ventricular systole occurring at the nont 
the radial tracing. It will be observed that this small wave occurs too early, and is followed by a pause longer than the pauses after the other pulse beats. In the jugular tracing the wave $c^{\prime}$ is due to the carotid, inasmuch as its appearance corresponds exactly with that of the small beat $x$ in the radial. The wave $a^{\prime}$ is due to the auricle, for the interval between it and the preceding auricular wave, $a$, exactly corresponds to the intervals separating all the other auricular waves. This retention of the rhythmicity of the auricle is one of the most striking features in this form of irregular heart, and the interpretation here given has been abundantly confirmed by other observers, and by experiments on animals. This tracing demonstrates, therefore, that here, as in Fig. I, the ventricle has contracted before the auricle. Engelmann has stated that the stimulus coming down from the auricle $\left(a^{\prime}\right)$ finds the ventricle refractory after its premature contrac. tion $\left(c^{\prime}\right)$, so that it fails to respond to the stimulus from $a^{\prime}$, but remains quiescent until the next physiological stimulus arrives from the auricle. This explanation may need modification, for here it is not that the ventricle is too exhausted, but, as this tracing demonstrates, it is because the conductivity of the $a v$ fibres is so exhausted that the stimulus from the auricle $\left(a^{\prime}\right)$ is not conveyed to the ventricle. This is brought out in the diagram (Fig. 3) representing the events in Fig. 2.

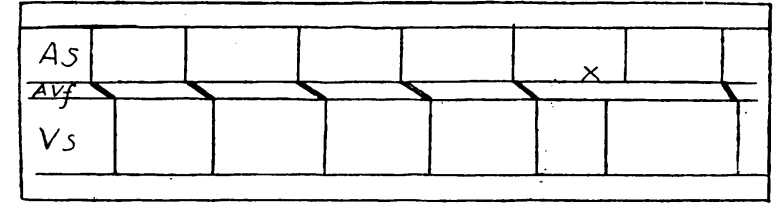

Fig. 3- - Diagram representing the events in Fig. 2. At $\times$ the stimulus from the following $A_{s}$ is blocked at the $a-v$ fibres.

To prove this statement consider the duration of the $a \cdot c$ intervals. All these intervals up to the extra-systole show a slight increase over the normal interval. Thus space $A$ in Fig. 2 equals ${ }^{3}$ of a second, while $\mathbf{A}^{\prime}$ equals about half that time. Tne salient point to be elucidated here is why is $\mathrm{A}^{\prime}$ so much shortened? If the $a \cdot v$ fibres had responded to. the stimulus from $a^{\prime}$ there would not have been a longer rest for these fibres, and the space $A^{\prime}$ would have had the same length as the other $a-c$ intervals. If, however; these fibres were stimulated in the production of the extra-systole $\left(c^{\prime}\right.$ and $\left.X\right)$, then they would be refractory to the stimulus from the auricle $\left(a^{\prime}\right)$ and would remain quiescent till the physiological stimulus arrived from the next auricular systole. In this manner they would have had a longer rest, their conductivity would be more completely restored, the stimulus would be conveyed across to the ventricle at a greater rate, and a shortening of the $a c$ interval would result, which shortening we find in $A^{\prime}$.

The next question is, Did the stimulation of these fibres precede or follow the ventricular extra-systole? I am of opinion that they preceded, and therefore caused, the ventricular extra-systole, because, first, the shortening of $A^{\prime}$ implies that the stimulation of the $a-v$ fibres must have taken place at a time considerably in advance of $a^{\prime}$; and secondly, it these fibres had been stimulated by a backward wave from the ventricle, the stimulus would have passed back to the auricle and interfered with the auricular rhythm. We see, however, that the auricular rhythm is undisturbed. But it might be asked, if the stimulus arose in the $a \cdot v$ fibres, why did it not pass backwards to the auricle as well as forwards to the auricle? In many cases it does so, as one can tell from the disturbed auricular rhythm. in other cases, as here, a minimal stimulus that will provoke a for ward contraction is n)t strong enoush to start one in the backward direction. Tais argument, if not concluvive, is at all events suggestive, a d indicates the lines which further investigation should pursue.

\section{The Results of Continuods Excitability of the} $a \cdot v$ Fibres.

Suppose, now, that instead of an occzsional extra stimulus, producing an occasional extra-systole, there was a continuous excitability producing a series of ventricular contractions, just as Gaskell produced it experimentally, how would such a change be manifested in the human subject? I have now collected abundant proof showing such results of extra stimulation. When this happens, the rhythm of the heart at once chang $\in \mathrm{s}$, producing either a verg rapid series of contractions, regalar or irregular (parexysmal tachycardia of ventricular origin), or a less rapid action of the heart, but with a continuously irregular rhythm. When this occurs a remarkable change takes place in the jugular pulse-a change from a pulse of the auricular type to one of the ventricular; that is, that whereas during the normal rhythm the auricular systole produced the principal wave in the jugular vein, there is during the abnormal rhythm no wave at this period of the cardiac cycle, but one large wave synchronous with and due to the ventricular systole. As a further proof I have found in a number of cases that patients with extra-systoles may show the irregular action at very varging intervals, occurring after every second or third beat. I have also found a few extrasystoles occurring together, and again have found a long. continued series of these, lasting cometimes for hours, sometimes for days and weeks, and in a number of cases persisting continuously until the heart became so exhausted that the patients succumbed. The proof that this rhythm was started by the ventricle $I$ have given in my book on the pulse, and with greater detail in a paper published in the BRITISE Mrdical Journal of March $5 \mathrm{th}, 1904$.

The Treatment of Cases of Ventricular Rhythm due to OVER-eXCITABILITY OF THE $a \cdot v$ FIBREs.

As I have remarked, the objective should be the restoration of the heart to the normal rhythm, and this can only be done by diminishing the excitability of the $a v$ fibres I have tried a great variety of methods-rest in bed, ma:sage, Schott movements, and all manner of drugs, and pushed the drugs as far as I dared, and have quite failed to achieve the object I had in view. I have seen many cases revert to the normal rhythm, and I have fancied that when this happened it was due to the method of treatment employed, but continued observation showed that it was due to other causes. This experience is well illustrated in the case of paroxysmal tachycardia of ventricular origin (there is another and rarer form of paroxysmal tachycardia where the rhythm is auricular in origin). The fewer cases a doctor sees of this complaint the more confident is he of his power to subdue the attack. I have $n n w$ records of a large number of cases, some of whom have had ten to twenty attacks, and I can say with confidence that when the heart has reverted to its normal rhythm it was due to some factor altogether outside my power to control.

In the paper in this series on the effect of digitalis on the conductivity of the $a-v$ fibres I showed how this drug readily and speedily affected this function in predisposed cases. Apart from such cases, I find that these fibres are the most resistant of all the muscle fibres of the heart to the influence of this drug, and when they are excitable the drug has but a slight effect upon their irritability, even when pushed as far as one dare. In many of my cases it has been absolutely without effect, and the following instance illustrates that. though it may have a slight effect, it fails to restore the normal rhythm.

In my paper on ventricular rhythm in the British Medical Journar of March 5 th, r904, I cited the case of a woman, 57 years of age, who had had numerous attacks of paroxysmal tachycardia, and I gave a number of tracings (Figs. 5 and $x_{3}$ to $2 \mathrm{r}$ ) showing how for years she had had single ventricular extra-systoles, and that these became more frequent, ultimately culminating in attacks of paroxysmal tachycardia, which lasted at times for hours, days, and weeks, and how these attacks would subside independent of any method of treatment. Since the last note published of that patient in November, $\mathrm{x}_{903}$, she has had a number of other attacks, one of which began while I was taking tracings of her pulse, and the jugular pulse at once changed from one of the auricular type to one of the ventricular. On October xst, r904 she was taken with an attack, which has continued up to the preseus (March, 1905), and since this attack started sho has been in an extremely feeble state, with dusky countenance, persistent cough, enlarged liver, and swollen legs, the heart enlarged, invariably rapid, and irregular (Figs. 4 and 5 ).

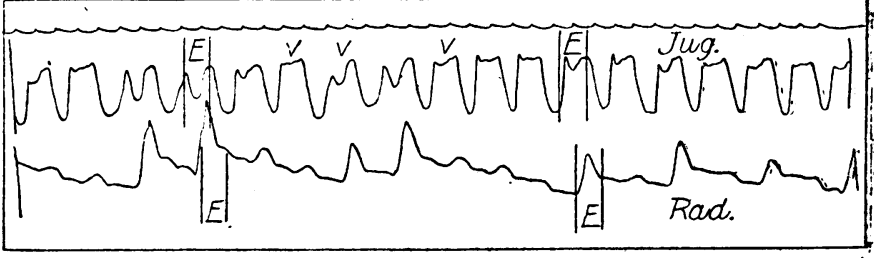

Fig. 4.-Weak, rupid, and irregular pulse due to the inception of the rhythm of the heart by the ventricle. The jugular pulse is of the ventricular type. Previcus to the attack the jugular pulse
resemkled Fig. $x$. (P. 120.) 


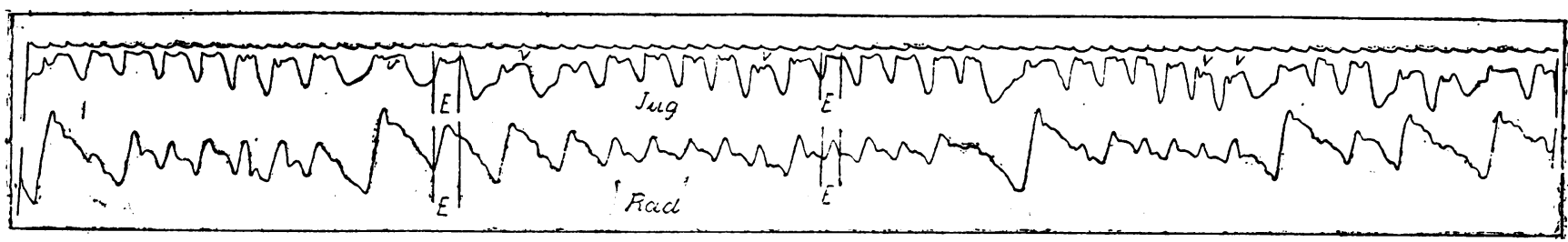

Fig. 6.-Small, rapid, and irregular pulse during an attack of heart failure. The rhythm lof the heart is ventricular. (February 6th, x905.)

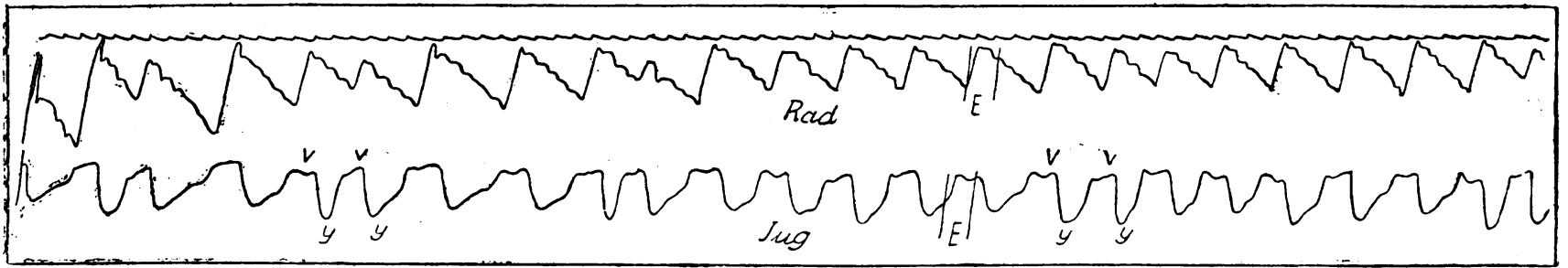

Fig. 7.-Shows the improvement in the pulse after digitalis. The jugular pulse shows that the rhythm of the heart is still ventricular. (February r7th, r905.)

By November rst, r904, her condition had become so bad, and as rest, treatment, and careful nursing had failed to afford any relief, I determined carefully to push the digitalis, which, in the form of tincture, she began to take on November ist $3 j$ per diem. No effect was produced till November 5 th, when she began to suffer from severe epigastric pain. This pain gradually descended lower in the central areas of the abdomen This pain gradually descended lower in the central areas of the abdomen
(umbilical and hypogastric), and when over the pubes terminated with en abundant fluid evacuation. She had several attacks like this on November 5 th and 6 th. The effect on the heart was to cause an appreciable slowing (Fig. 5), the rhythm, however, remaining ventricular.

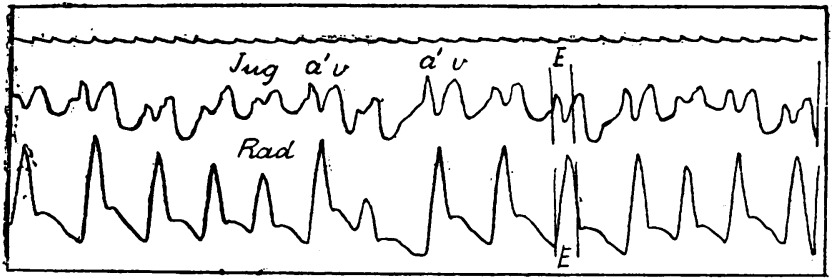

Fig. 5.-Shows effect of digitalis on the pulse. The rate is slower (ro8 per minute), but the ventricular rhythm persists.

The patient did not experience at first much relief. The digitalis was stopped, and two days later she experienced decided benefit, the breathing being easier and the legs less swollen, though the quantity of urine passed was not much increased-about $20 \mathrm{Oz}$. per diem. Ten days later she got gradually worse, and I tried her again with digitalis, but one dose of ro minims of the tincture immediately produced such a sensation of sinking and of pain in the epigastric region that she asserted she would rather die than take another dose. Since that time till now (March, 1905), she has been practically bedridden, the heart still acting weakly and irregularly.

The digitalis had an effect on the heart muscle, but the musculature of the digestive tract was evidentjy more susceptible than the heart muscle, and the disturbing effect on the stomach and bowels probably prevented sufficient of the drug boing taken to affect materially the heart muscle. Although digitalis may fail to restore the normal rbythm, it may have a beneficial effect on certain cases of ventricular rhythm. Thus a woman, aged 57, who has been under my care for two years, invariably has attacks of heart failure. when she ceases to take the digitalis. I have tried strophanthus and other drugs, but with no good result. The last attack was on February 6th, rg05, with dropsy, enlarged liver, blue face, great pulsation in the veins of the neck, and scanty urine. The pulse was small, rapid, and very irregular (Fig. 6).

She was prescribed tincture of digitalis ro minims three times a day She began to improve at once, and by November 17 th the dropsy had nearly all disappeared; she breathed easier and passed more urine. The pulse became slower and more regujar, but the ventricular rhythm persisted, and the jugular pulse remained very large (Fjg. 7). A year ago, after a course of digitalis, even the jugular pulse would disappear, tricular in origin.

\section{The Production of Ventricular Extra-systoles by} Digitalis.

In the last paper of this series I gave a tracing showing extra-systoles occurring after digitalis. In another recent case I observed a similar occurrence. A female, aged 22, complained of being weak, of having attacks of palpitation, of having fainted; : she had a presystolic murmur. On February roth, 1905, she was prescribed tincture of digitalis. In a few days she felt distinctly better. On the I th, after taking 3 iv of the tincture, she complained of distressing "bumping" of her heart. The pulse felt regularly intermittent, and a tracing showed the regular occurrence of ventricular extra-systoles after every second normal beat (Fig. 8).

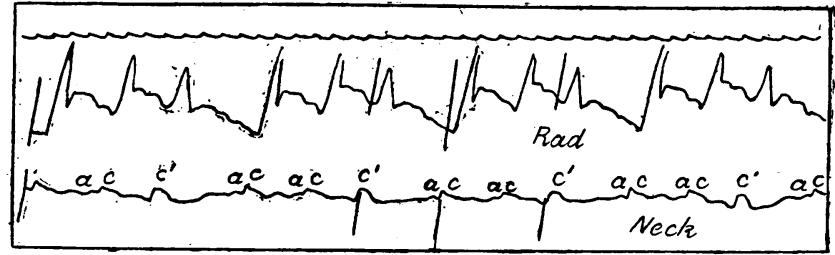

Fig. 8.-The radial pulse occurs in groups of three beats: the last of the three beats occurs a lintle earlier than the second beat. The faint tracing from the neck shows the carotid wave $c^{\prime}$, due to the ventricular extra-systole, always a little larger than $c$, due to the fact that at this time lie auricular wave also appeared in the
jugular pulse and assisted to raise the lever.

The digitalis was stopped, and the next day all trace of extrasystoles had disappeared. She began again on the 20 th to take to minims twice daily, and on the 24th the "bumping" of her heart began again, and a tracing showed the same arrhythmia as Fig. 7. The drug was stopped; there has been no recurrence of the irregularity, and the patient's condition is distinctly improved.

As digitalis when pushed has a depressing influence on all the functions of the heart, it seems at first sight strange that it should manifest a tendency to produce extra-systoles, which implies a certain degree of irritability. I can only account for it by the fact that while it has depressed the functional activity of all the other fibres of the heart, as shown by the slowing, it has failed to affect the $a \cdot v$ fibres, which retain their previous irritability, and "fire off" an extra-systole from time to time. But why it should do this so regularly I do not understand. If this surmise is right, it forms an additiona argument in favour of the $a \cdot v$ fibres being the source of the extra-systoles.

Medical Oertificates for Marriage.-It is said that the Armenian Church in the Caucasus has decided that no priest shall be allowed to perform the marriage ceremony unless the contracting parties can produce a medical warranty of physical soundness.

Brouests to Medical Charitirs.-Under the will of the late Mr. Henry Allen of Bolney, 8ussex, which has now been proved, St. Mark's Hospital for Fistula receives a sum of fl,coo, the London Fever Hospital half that amount, and 8t. Mary's Hospital, Paddington, and the Brompton Cancer Hospital $£ 3 c 0$ each, while $£ 100$ goes to the Dorking Cottage Hospital. After the payment of certain other legacies it is expected that there will be a residue of $f 10,000$, which sum is left in trust for such charitable institutions as the trustees may themselves select, the desire being expressed that some portion may be allotted to charities connected with Great Miseenden, Buckinghamshire. 